\title{
Recurrent disease in 25 patients with acquired thrombotic thrombocytopenic purpura and hemolytic uremic syndrome during 6.5 years follow-
}

up

\begin{abstract}
Thrombotic thrombocytopenic purpura (TTP) and hemolytic uremic syndrome (HUS) are manifestations of excessive microvascular platelet aggregation and present dangerous thrombotic microangiopathies (TMA). The clinical presentation of TTP/HUS is variable in terms of type and severity of clinical symptoms. Before the introduction of plasma exchange (PEX) for the treatment of acute TTP episode mortality reached $90 \%$, but decreased to $8-30 \%$ following the adoption of PEX. Acute idiopathic TTP is the most common type of TTP seen in clinical practice. The clinical course of idiopathic TTP is characterized by recurrent disease episodes in up to $50 \%$ of cases; with a higher risk of recurrence in those with severe deficiency of the von Willebrand factor cleaving-protease ADAMTS13 during acute episodes. We report on the recurrences among 25 patients with TTP and HUS, in whom we monitored routine laboratory parameters, response to treatment as well as ADAMTS13 activity and antiADAMTS13 antibodies in acute phase and remission.
\end{abstract}

Volume 4 Issue 2 - 2017

Vucelic D,' Mikovic D'
'Clinic of Digestive Surgery, Clinical Centre of Serbia, Serbia
${ }^{2}$ Blood Transfusion Institute of Serbia, Serbia

Correspondence: Dragica Vucelic, Clinic of Digestive Surgery, Clinical Center of Serbia, Belgrade, Serbia, Koste Todorovic St. 6, I I.000 Belgrade, Serbia, Fax +38 I (I I) 3065967, Tel +38 I (I I) 3663757, Email vucelicd@gmail.com

Received: February 13, 2017 | Published: April 10, 2017

Keywords: ADAMTS13, recurrence, relapse, von willebrand factor, remission

Abbreviations: TTP, thrombotic thrombocytopenic purpura; HUS, hemolytic uremic syndrome; TMA, thrombotic microangiopathies; PEx, plasma exchange; LDH, lactate dehydrogenase; BNU, blood urea nitrogen; CNS, central nervous system; FFP, fresh frozen plasma; p.v.e., plasma volume exchange; $\mathrm{Pl}$, platelets; $\mathrm{Hb}$, hemoglobin; Re, reticulocytes; $\mathrm{Cr}$, creatinine

\section{Introduction}

Acquired thrombotic thrombocytopenic purpura (TTP) and hemolytic uremic syndrome (HUS) remain a intriguing diseases not only because of rarity and difficult diagnosis, but also due to uncertainty of disease nature with regard to the risk for relapse. The frequency of relapse is different among different categories of TTP and HUS. Patients who survive the first acute episode of the TTP may remain asymptomatic for the rest of their life. ${ }^{1}$ Relapses are rare among patients with secondary TTP and those who had a prodrome of bloody diarrhea. Among patients with acquired atypical HUS, recurrent disease occurs in those with an immune-mediated druginduced syndrome with repeated ingestion of drug while the others usually have a single episode. ${ }^{2-4}$ In many patients who have acquired idiopathic TTP associated with ADAMTS13 (A Disintegrin and Metalloproteinase with thrombospondin motif-13) deficiency there is the presence of transient, or intermittently recurrent, defect in immune regulation. ${ }^{4}$ Idiopathic TTP caused by ADAMTS13 deficiency tends to relapse. In majority of cases, relapses are associated with persistent or recurrent severe protease deficiency which is almost totally restricted to patients who have idiopathic TTP. ${ }^{5}$ Recurrent episodes of disease occur in up to $50 \%$ of patients who survive their initial episode of TTP., ${ }^{3,5-7}$ Relapses usually develop during the first year after the initial episode and most patients will have only one relapse. ${ }^{2}$
Furthermore, around one third of all patients with acquired TTP have a chronic course with multiple relapses. ${ }^{7,8}$ In the absence of reliable indicators of the long-term clinical outcome, the prediction of which patients who achieve complete remission of acute episode will recur is not possible. ${ }^{9}$ Current data suggest that the risk of recurrences being higher in patients with severe ADAMTS13 deficiency and anti-ADAMTS13 auto antibodies during acute episodes. ${ }^{10-14}$ The cumulative risk of recurrence at 7.5years after the first episode in patients with ADAMTS13 activity below $10 \%$ at presentation was estimated to be $41 \%$, 10 times that of patients with activity above $10 \%$ (4\% risk at 7.5 years). ${ }^{10}$ Persistence of ADAMTS13 deficiency and of auto antibodies during disease remission is also associated with increased risk of recurrence. ${ }^{15,16}$ We report on the recurrences among 25patients with TTP and HUS, in whom we monitored routine laboratory parameters, response to treatment as well as ADAMTS13 activity and anti-ADAMTS13 antibodies in acute phase and remission.

\section{Patients and definition of clinical categories}

We evaluated for recurrence in 28 patients with clinical and laboratory findings that suggested a diagnosis of acquired TTP and HUS. Clinical presentation was defined with idiopathic TTP in 18, secondary TTP in 4, diarrhea positive HUS (D+ HUS-typical HUS) in 3 and diarrhea negative HUS (D- HUS-atypical HUS) in 3 patients. Of 28,25 patients survived acute episode. Survival rate was $89.3 \%$. Three patients (idiopathic TTP, secondary TTP with underlying progressive malignancy and D+ HUS associated with tacrolimus intake) died during the initial hospitalization. Among survivors with secondary TTP, syndrome was associated with connective tissue systemic disease (patient $\mathrm{n}^{0} 18$ ), syndrome sicca (patient $\left.\mathrm{n}^{0} 19\right)$ and systemic lupus erythematosus-SLE (patient $\mathrm{n}^{0} 20$ ). One patient with D- HUS 
exhibited onset of disease in the context of SLE (patient $\mathrm{n}^{0} 24$ ) and one of D+ HUS patients was treated with tacrolimus. Among survivors, $22 / 25$ presented with their initial TTP or HUS episode, whereas $3 / 25$ patients (cases $n^{0} 5,13$ and 16 with idiopathic TTP) presented at our observation on their first relapse after 11, 7 and 6years from initial episode. There were $20 \mathrm{female}(80 \%)$ and 5 male $(20 \%)$ patients. The ages ranged from 17 to 68 years.

\section{Diagnosis of acute TTP and HUS}

Diagnosis of acute TTP was established when at least two of the following criteria were met: thrombocytopenia with no apparent cause, Coombs-negative hemolytic anemia with schistocytes in the blood film, high serum levels of lactate dehydrogenase (LDH) and signs and symptoms compatible with organ ischemia. ${ }^{15}$

\section{Complete remission}

Complete remission was defined as no plasma exchange treatment at least 30 days after laboratory and clinical normalization: normal platelet count for 2 consecutive days, recovery of LDH and haptoglobin to normal, normalization of organs function with a rising hemoglobin. , $11,12,17^{2}$

\section{Relapse}

Relapse was defined as the re-emergence of clinical symptoms and/ or laboratory criteria compatible with a diagnosis of TTP occurring at least 30 days or later after remission of the acute episode. ${ }^{15}$

\section{Measurement of ADAMTS I 3 activity}

The ADAMTS13 activity was measured at the Angelo Bianchi
Bonomi Haemophilia and Thrombosis Centre in Milan, Italy. Protease plasmatic activity was assayed using collagen binding method on citrated plasma as previously described. ${ }^{15}$ ADAMTS 13 activities were arbitrarily divided into four categories: 1 . less than $6 \%$ which is the detection limit of the assay (severe deficiency); 2 between $6 \%$ and $25 \%$ (moderate deficiency); 3 between $26 \%$ and $46 \%$ (mild deficiency); 4 greater than $46 \%$ (normal).

\section{Anti-ADAMTSI 3 antibodies testing}

Anti-ADAMTS13 antibodies were detected by western blot technique, as previously reported..$^{15}$

\section{Clinical presentation, treatment and results of ADAMTSI 3 testing in survivors}

Among 25 survivors there were 17patients with idiopathic TTP, 3 with secondary TTP, 2 with D+HUS and 3 with D- HUS. During follow up period lasting up to 6.5 years, none of the patients with secondary TTP, D+ HUS and D- HUS recurred. Recurrences were manifested in $7 / 17(41 \%)$ surviving patients with idiopathic TTP. Three of them, were seen in their first relapse (cases $n^{0} 5,13$ and 16) 11, 7 and 6years from original episode. During the follow up period dated from the entry in this study, five patients with idiopathic TTP (cases $n^{0} 3,4,7$, 13 and 14) had relapses (29\%). Of them, relapses were seen in 3 of the 13 female and in 2 of the 4 male patients. The duration of follow-up of these patients has ranged from 1year and 4months to 5years and 4months. Table 1 provides the results on the ADAMTS13 activity and anti-ADAMTS13 antibodies in acute episode and remission, duration of follow up period and occurrence/number of relapse for patients who survived the initial episode $(\mathrm{N}=25)$.

Table I Results of ADAMTSI 3 activity and anti-ADAMTSI 3 antibodies in acute episode and remission $(\mathrm{N}=25)$

\begin{tabular}{|c|c|c|c|c|c|c|c|c|c|}
\hline $\begin{array}{l}\text { Patient } \\
\mathbf{N}^{0}\end{array}$ & $\begin{array}{l}\text { Agel } \\
\text { Sex }\end{array}$ & $\begin{array}{l}\text { Clinical } \\
\text { Type TTPI } \\
\text { HUS }\end{array}$ & $\begin{array}{l}\text { ADAMTSI3 } \\
\text { Acute } \\
\text { Episode }\end{array}$ & $\begin{array}{l}\text { Anti- } \\
\text { ADAMTSI } 3 \\
\text { Acute } \\
\text { Episode }\end{array}$ & $\begin{array}{l}\text { ADAMTSI } 3 \\
\text { Remission }\end{array}$ & $\begin{array}{l}\text { Anti- } \\
\text { ADAMTSI } 3 \\
\text { Remission }\end{array}$ & $\begin{array}{l}\text { Follow- } \\
\text { up After } \\
\text { Entry to } \\
\text { Study }\end{array}$ & $\begin{array}{l}\text { Relapse } \\
\text { After } \\
\text { Entry to } \\
\text { Study }\end{array}$ & $\begin{array}{l}\text { Episode } \\
\text { Before Entry } \\
\text { to Study }\end{array}$ \\
\hline I & $55 \mathrm{~F}$ & Idiopathic & $24 \%$ & Negative & Not done & Not done & 6y $5 \mathrm{~m}$ & - & - \\
\hline 2 & $27 \mathrm{~F}$ & Idiopathic & $40 \%$ & Negative & $100 \%$ & Negative & $5 y \mathrm{IIm}$ & - & - \\
\hline 3 & $27 M$ & Idiopathic & $<6 \%$ & Positive & $13 \%$ & Positive & $5 y 5 m$ & yes (I) & - \\
\hline 4 & $45 \mathrm{~F}$ & Idiopathic & $37 \%$ & Positive & $75 \%$ & Positive & $5 y 3 m$ & yes (I) & - \\
\hline 5 & $45 \mathrm{~F}$ & Idiopathic & II\% & Negative & $20 \%$ & Negative & $3 y 6 m$ & - & $\begin{array}{l}\text { yes- I (I ly } \\
\text { ago) }\end{array}$ \\
\hline 6 & $33 \mathrm{~F}$ & Idiopathic & $<6 \%$ & Positive & $30 \%$ & Positive & $3 y 5 m$ & - & - \\
\hline 7 & $5 I M$ & Idiopathic & $<6 \%$ & Positive & $<6 \%$ & Positive & $3 y 5 m$ & yes (2) & - \\
\hline 8 & $39 \mathrm{~F}$ & Idiopathic & $30 \%$ & Positive & $83 \%$ & Positive & $2 y 3 m$ & - & - \\
\hline 9 & $25 \mathrm{~F}$ & Idiopathic & $<6 \%$ & Positive & $36 \%$ & Positive & $2 y 3 m$ & - & - \\
\hline 10 & $2 I M$ & Idiopathic & $<6 \%$ & Positive & $<6 \%$ & Positive & Iy $10 \mathrm{~m}$ & - & - \\
\hline II & $30 M$ & Idiopathic & $<6 \%$ & Positive & $9 \%$ & Positive & ly $5 \mathrm{~m}$ & - & - \\
\hline 12 & $34 \mathrm{~F}$ & Idiopathic & $<6 \%$ & Positive & $54 \%$ & Positive & ly $3 \mathrm{~m}$ & - & - \\
\hline 13 & $48 \mathrm{~F}$ & Idiopathic & $<6 \%$ & Positive & $160 \%$ & Negative & ly $5 \mathrm{~m}$ & yes (3) & yes- I (7y ago) \\
\hline 14 & $17 \mathrm{~F}$ & Idiopathic & $<6 \%$ & Positive & $61 \%$ & Positive & $2 y \mathrm{Im}$ & yes (2) & - \\
\hline 15 & $41 \mathrm{~F}$ & Idiopathic & $<6 \%$ & Positive & $55 \%$ & Positive & $2 y \mathrm{Im}$ & - & - \\
\hline 16 & $33 \mathrm{~F}$ & Idiopathic & $<6 \%$ & Positive & $97 \%$ & Positive & $2 y \mathrm{Im}$ & - & yes- I (6y ago) \\
\hline
\end{tabular}


Table Continued....

\begin{tabular}{|c|c|c|c|c|c|c|c|c|c|}
\hline $\begin{array}{l}\text { Patient } \\
\mathbf{N}^{0}\end{array}$ & $\begin{array}{l}\text { Agel } \\
\text { Sex }\end{array}$ & $\begin{array}{l}\text { Clinical } \\
\text { Type TTP/ } \\
\text { HUS }\end{array}$ & $\begin{array}{l}\text { ADAMTSI } 3 \\
\text { Acute } \\
\text { Episode }\end{array}$ & $\begin{array}{l}\text { Anti- } \\
\text { ADAMTSI3 } \\
\text { Acute } \\
\text { Episode }\end{array}$ & $\begin{array}{l}\text { ADAMTSI3 } \\
\text { Remission }\end{array}$ & $\begin{array}{l}\text { Anti- } \\
\text { ADAMTSI } 3 \\
\text { Remission }\end{array}$ & $\begin{array}{l}\text { Follow- } \\
\text { up After } \\
\text { Entry to } \\
\text { Study }\end{array}$ & $\begin{array}{l}\text { Relapse } \\
\text { After } \\
\text { Entry to } \\
\text { Study }\end{array}$ & $\begin{array}{l}\text { Episode } \\
\text { Before Entry } \\
\text { to Study }\end{array}$ \\
\hline 17 & $30 \mathrm{~F}$ & Idiopathic & $<6 \%$ & Positive & $93 \%$ & Negative & Iy Im & - & - \\
\hline 18 & $23 \mathrm{~F}$ & Secondary & $13 \%$ & Negative & $106 \%$ & Negative & $6 y 6 M$ & - & - \\
\hline 19 & $68 \mathrm{~F}$ & Secondary & $14 \%$ & Positive & $65 \%$ & Negative & $3 y 10 \mathrm{~m}$ & - & - \\
\hline 20 & $36 \mathrm{~F}$ & Secondary & $<6 \%$ & Positive & $151 \%$ & Negative & $2 y 2 m$ & - & - \\
\hline 21 & $52 \mathrm{~F}$ & $D+H U S$ & $52 \%$ & Negative & $83 \%$ & Negative & $6 y \mathrm{Im}$ & - & - \\
\hline 22 & $62 \mathrm{~F}$ & $D+$ HUS & $54 \%$ & Negative & $95 \%$ & Negative & $5 y \mathrm{Im}$ & - & - \\
\hline 23 & $28 \mathrm{~F}$ & D- HUS & $37 \%$ & Negative & $77 \%$ & Negative & $2 y 4 m$ & - & - \\
\hline 24 & $20 \mathrm{~F}$ & D- HUS & $42 \%$ & Negative & $135 \%$ & Negative & $2 y 3 m$ & - & - \\
\hline 25 & $32 M$ & D- HUS & $64 \%$ & Negative & $97 \%$ & Negative & $2 y \| \mathrm{Im}$ & - & - \\
\hline
\end{tabular}

F: Female, M: Male; m: month; y: year

ADAMTSI 3 results are referred to acute phase and remission related to first relapse in cases $n^{0} 5, I 3$ and $I 6$. For remaining patients, results are referred to acute phase and remission related to their initial episodes. Patient $n^{0} \mathrm{I}$ was tested only in acute phase of initial episode.

In acute phase, in the subgroup of patients with idiopathic TTP $(\mathrm{N}=17)$ severe deficiency was determined in $12(70.58 \%)$, moderate deficiency in $2(11.76 \%)$ and mildly reduced activity in $3(17.64 \%)$ patients. Specific antibodies were found in plasma of all patients with severe ADAMTS 13 deficiency, in none of the 2patients with moderately, but they were present in plasma of $2 / 3$ patients with mildly reduced protease level. In remission, severe protease deficiency was detected in $2(11.76 \%)$, moderate deficiency in $3(17.64 \%)$, mild deficiency in $2(11.76 \%)$ and normal activity in $9(52.94 \%)$ patients while in one patient (5.88\%) testing has not been done. Among the 16 tested patients, antibodies were present in 12 of them with frequency as follows: in 2 with severe, $2 / 3$ with moderate, 2 with mild deficiency as well as in $6 / 9$ patients in whom protease activity was normalized. In acute phase of secondary TTP, in the category of severe protease deficiency there was $1 / 3(33.33 \%)$ and in the category of moderate reduced protease activity there were $2 / 3(66.66 \%)$ patients. Antibodies were detected in plasma of patient with SLE-associated disease with severe ADAMTS13 activity and in one with syndrome sicca related form with moderately reduced protease activity. In patient with TTP secondary to connective tissue disease, antibodies were not present. In remission, antibodies were absent in all patients.

A mildly reduced ADAMTS13 activity was found in acute episode of $2 / 3(66.66 \%)$ patients with D- HUS. Normal protease levels were measured only in the cases with HUS (all patients with D+ HUS and $1 / 3$ patients with D- HUS). In samples of 2 patients with D- HUS and mildly reduced ADAMTS13 activity antibodies were absent. None of the patients with normal protease activity had antibodies. In remission, protease activity normalized in 2patients with D- HUS with reduced activity in acute phase. Among three cases with fatal outcome, it had been shown that patient with idiopathic TTP had auto immune severe deficiency of ADAMTS13. She presented with coma and died on admission day before plasma exchange (PEX) treatment could be organized. In female patient with TTP secondary to malignancy, ADAMTS13 activity was moderately reduced $(25 \%)$ in absence of antibodies. ADAMTS13 activity was normal (53\%) in male patient defined as having typical HUS with prodromal diarrhea.

\section{Description of patients who experienced recurrences}

We saw 3/17 patients with idiopathic TTP in their first relapse which were manifested several years after initial episode. In one patient, we have seen first relapse as late as 11years after initial episode. One of these 3patients relapsed two times in a follow-up period after entry to this study. Including these patients, in all there have been $7 / 17(41,17 \%)$ idiopathic TTP patients with recurrent disease. It is likely that some our idiopathic TTP patients will recur in the future, since 10 of them are only on the 1 to 3year follow up period. We present 7 patients who experienced recurrences as well as two patients have been considered interesting because in a follow up period after initial episode of idiopathic TTP they had uncomplicated pregnancy and delivery each. Table 2 shows presenting features and Table 3 describes treatment of patients with recurrences at study entry $(\mathrm{N}=7)$. In all patients $(\mathrm{N}=28)$ included in this cohort, ADAMTS13 parameters were not known at the time of patient's treatment. Our standard clinical practice was institution of PEX/fresh frozen plasma (FFP) in combination with corticosteroids in all patients, with adding additional therapies in cases with poor/delayed clinical response during the course of treatment.

Patient $n^{0} 3$ a 27 -year old previously healthy male, obese (weight $106 \mathrm{~kg}$, height $180 \mathrm{~cm}$ ) experienced initial episode with no apparent trigger event. On presentation, he had purpura, lumbal pain, platelet count $20 \times 10^{9} / \mathrm{L}$, hemoglobin $93 \mathrm{~g} / \mathrm{L}$, LDH $1372 \mathrm{IU} / \mathrm{L}$, reticulocytes $17 \%, 5$ schistocytes in blood film and microhematuria. Complete remission was achieved by 8 double volume daily PEX with concomitant corticosteroid administration (methylprednisolone 1g/ $\mathrm{kg}$ iv. for 3 days, than prednisolone per os $1 \mathrm{mg} / \mathrm{kg}$ ). Vincristine at a dosage of $1 \mathrm{mg}$ was given at day 8 in addition to PEX and $1 \mathrm{mg}$ at day 12. Consolidation treatment consisted of 2 PEX session followed by FFP transfusion in dose $15 \mathrm{~mL} / \mathrm{kg}$ for 3days. ADAMTS13 activity was severe reduced in acute phase and remained at low level (13\%) in presence of antibodies in remission. After 3 years he experienced a relapse. First relapse treated successfully with standard therapy which consisted of 6 daily double volume PEX and corticosteroids. 
Table 2 Presenting features at study entry of patients who had relapses of TTP $(\mathrm{N}=7)$

\begin{tabular}{|c|c|c|c|c|c|c|c|c|}
\hline \multirow{2}{*}{ Patient } & \multirow{2}{*}{$\begin{array}{l}\text { Previous } \\
\text { episode }\end{array}$} & \multicolumn{7}{|c|}{ Presenting features at study entry } \\
\hline & & PI XI09/L & Hb g/L & LDH IU/L & $\operatorname{Re} \%$ & $\mathrm{Cr} / \mathrm{BNU} \mu \mathrm{mol} / \mathrm{L} / \mathrm{mmol} / \mathrm{L}$ & CNS Abnormalities & Fever \\
\hline 3 & No & 20 & 93 & 1372 & 17 & $102 / 7.6$ & - & - \\
\hline 4 & No & 50 & 93 & 643 & 15 & $83 / 4.1$ & Headache, Dizziness & - \\
\hline 5 & Yes & 28 & 92 & 2929 & 7 & $183 / 24$ & - & Yes \\
\hline 13 & Yes & 19 & 73 & 2797 & 16.6 & $96 / 9.3$ & Epileptic Attack & - \\
\hline 14 & No & 12 & 103 & 2795 & 9.3 & $67 / 10.1$ & Headache, Aphasia, Unconsciousness & - \\
\hline 16 & Yes & 80 & 115 & 783 & 5.6 & $62 / 3.8$ & - & Yes \\
\hline
\end{tabular}

Table 3 Treatment of patients with recurrences at study entry $(N=7)$

\begin{tabular}{|c|c|c|c|c|c|}
\hline \multirow{2}{*}{ Patient } & \multicolumn{2}{|c|}{ PEX/FFP treatment } & \multirow{2}{*}{$\begin{array}{l}\text { Consolidation treatment } \\
\text { PEX/FFP }\end{array}$} & \multirow{2}{*}{ Steroid therapy } & \multirow{2}{*}{ Additional immune therapy } \\
\hline & PEX No/p.v.e & FFP & & & \\
\hline 3 & 8/double & & $\begin{array}{l}2 \text { double volume PEX } \\
15 \mathrm{ml} / \mathrm{kg} 3 \mathrm{~d}\end{array}$ & $\begin{array}{l}\text { Metylprednisolone Ig 3d } \\
\text { prednisone Img/kg }\end{array}$ & Vincristine $2 \mathrm{mg}$ \\
\hline 4 & 5/double & & $-/ 15 \mathrm{ml} / \mathrm{kg} 3 \mathrm{~d}$ & $\begin{array}{l}\text { Metylprednisolone Ig 3d } \\
\text { predniisone I mg/kg }\end{array}$ & - \\
\hline 7 & 5/double & & $-/ / 5-20 \mathrm{ml} / \mathrm{kg} 4 \mathrm{~d}$ & $\begin{array}{l}\text { Metylprednisolone Ig 3d } \\
\text { prednisone Img/kg }\end{array}$ & - \\
\hline 13 & I0/single $+5 / 1.5$ & & - & $\begin{array}{l}\text { Metylprednisolone Ig 3d } \\
\text { prednisone Img/kg }\end{array}$ & $\begin{array}{l}\text { Vincristine } 4 \mathrm{mg} \\
\mathrm{IV} \lg 0.4 \mathrm{~g} / \mathrm{kg} 5 \mathrm{~d}\end{array}$ \\
\hline 14 & $5 /$ single $+16 / 1.5$ & & $\begin{array}{l}2 \text { single volume PEX } \\
800 \mathrm{ML} / \text { day } 4 \mathrm{~d}\end{array}$ & $\begin{array}{l}\text { Metylprednisolone } \\
500 \mathrm{mg} \text { Id }+ \text { Ig } 2 \mathrm{~d} \\
\text { prednisone } 2 \mathrm{mg} / \mathrm{kg}\end{array}$ & IV Ig 20g/day 2 d \\
\hline 16 & - & I5ML/kg 3d & - & $\begin{array}{l}\text { Metylprednisolone Ig 3d } \\
\text { prednisone } \mathrm{Img} / \mathrm{kg}\end{array}$ & - \\
\hline
\end{tabular}

Patient $n^{0} 4$, a 45 -year old female, had first episode following a 10-days history of an upper respiratory tract infection. Clinically, she presented with prolonged gynecological bleeding, purpura, headache and dizziness. She responded with complete remission to 5 double volume PEX and concomitant corticosteroid therapy. She had first relapse $3 y e a r s$ and 10 months after initial episode. Recurrence had been triggered by flu. Laboratory examination revealed severe thrombocytopenia $\left(14 \times 10^{9} / \mathrm{L}\right)$ and hemolytic anemia (hemoglobin $112 \mathrm{~g} / \mathrm{L}, \mathrm{LDH} 1272 \mathrm{IU} / \mathrm{L}$ ), reticulocyte 5\%, schistocytes 3-4 in blood film and undetectable haptoglobin). Complete remission was achieved with 5 PEX procedures with 1.5 volume exchange and corticosteroid therapy. ADAMTS13 activity in acute phase of her initial episode was $37 \%$ in presence of antibodies, while in first remission protease activity normalized with the persistence of antibodies.

Patient $n^{0} 7$, 49-year old previously healthy male, presented with idiopathic TTP. Clinical course and response to PEX and corticosteroids were typical of primary TTP. The presenting symptoms and signs observed during the first episode included the development of fever (up to $38^{\circ} \mathrm{C}$ ), headache, weakness, fatigue, tachycardia, jaundice and a bleeding diathesis manifested as macrohematuria and hematomas at the venipuncture sites. Laboratory data at hospital admission were as follows: platelet count $16 \times 10^{9} / \mathrm{L}$, hemoglobin $65 \mathrm{~g} / \mathrm{L}, \mathrm{LDH}$ $1726 \mathrm{IU} / \mathrm{L}$, undetectable haptoglobin, reticulocytes $5 \%, 5$ schistocytes per high-power field in peripheral blood smear. He responded well to first line treatment consisted of once daily PEX procedure of double volume exchange using FFP and corticosteroids therapy with pulse methylprednisolone in dose $1 \mathrm{~g} / \mathrm{iv}$. for the first three days thereafter followed by per orally prednisone administration in dose $1 \mathrm{mg} / \mathrm{kg} /$ day. After cessation of daily PEX, consolidation treatment was performed with FFP in a dose of $15-20 \mathrm{ml} / \mathrm{kg}$ for four consecutive days. After 9months, in the absence of known trigger condition, patient relapsed but had no clinical manifestation and was diagnosed on the basis of abnormalities found during routine laboratory examinations (a combination of thrombocytopenia and a microangiopathic hemolytic anemia). He has been considered as having chronic form of TTP and was scheduled for splenectomy. While waiting for splenectomy, he was given with maintenance therapy with acetylsalicylic acid $100 \mathrm{mg} /$ day and prednisolone $1 \mathrm{mg} / \mathrm{kg} /$ day. Suddenly, subsequent relapse of thrombotic microangiopathy occurred with nausea and chest pain with propagation to left arm. Interestingly, laboratory findings were as follows: platelet count $63 \times 10^{\%} / 1$, hemoglobin $129 \mathrm{~g} / \mathrm{L}$, LDH $710 \mathrm{IU} / \mathrm{L}$, reticulocytes 3\%, 2 schistocytes per high-power field in peripheral blood smear and detectable haptoglobin $0.96 \mathrm{~g} / \mathrm{L}$. Patient was urgently transferred to Coronary unit where he was diagnosed with acute myocardial infarction based on the presence of chest pain, electrocardiographic alterations (ST elevation in D1, D2, AVL, V2$\mathrm{V} 5$, negative $\mathrm{T}$ in $\mathrm{V} 2-\mathrm{V} 4$ ) and elevated cardiac troponin $8.57 \mu \mathrm{g} / \mathrm{L}$ 
(normal $<0.1 \mu \mathrm{g} / \mathrm{L}$ ). Patient underwent specific cardiologic therapy and decision was made to commence PEX session without delay. Corticosteroids were considered to be contraindicated in the context of ongoing clinical setting. Complete remission was achieved upon administration of 6 PEX procedures (5 with double and 1 with single volume exchange). Consolidation treatment consisted of daily FFP transfusion in volume of $1500 \mathrm{~mL} /$ day for 6 days. At the time of complete clinical and laboratory remission defined with platelet count $206 \times 10^{\%} /, \mathrm{LDH} 418 \mathrm{IU} / \mathrm{L}$, haptoglobine $1.96 \mathrm{~g} / \mathrm{L}$ and stable hemoglobin, vincristine was given in 4 doses ( $1 \mathrm{mg}$ per dose). So far, patient is in stable remission. It was shown that patient belongs autoimmune TTP form with severe deficiency of ADAMTS13. In remission, ongoing autoimmune responses against ADAMTS13 persisted.

Patient $n^{0} 13$, who was reported previously,$^{18}$ was first seen when she was suffering the first episode of idiopathic TTP. Diagnosis of idiopathic TTP was made on clinical grounds. She remained refractory after 22 days of combined treatment with corticosteroids and PEX, which were performed by single volume exchanges for the first 7 days followed by intensifying regimen with twice daily PEX. Due to poor response to intensive treatment regime, on day 23 treatment was augmented with vincristine in two doses to a total dose of $2 \mathrm{mg}$. The patient achieved complete remission and remained well at follow-up for 7years. After this time period patient has experienced relapses. The clinical course of the first relapse was also typical of idiopathic TTP. Initial treatment consisted of single volume daily PEX against FFP and pulse methylprednisolone (1g/day iv.) for the first 3days from the presentation followed by $1 \mathrm{mg} / \mathrm{kg}$ of prednisolone. However, neither thrombocytopenia nor hemolysis improved and the clinical course deteriorated with epileptic attack development on day 3. During the course of the first 5 days of this episode, patient was given with 11 red blood cells units. Along with PEX with 1.5 volume exchanges (total number of PEX sessions was 15) and corticosteroids, additional therapy consisted of chemotherapy with vincristine (1mg on days 1 , 4, 7 and 10) and intravenous immunoglobulin (IV Ig) in a dose of $0.4 \mathrm{~g} / \mathrm{kg}$ for 5 consecutive days. Adjuvant therapy with vincristine and IV Ig was introduced on 5 and 6 day, respectively. After 19 days she could be discharged in complete clinical and laboratory remission (platelet count $268 \times 10^{9} / \mathrm{L}, \mathrm{LDH} 453 \mathrm{IU} / \mathrm{L}$ ). ADAMTS13 activity and anti-ADAMTS13 antibodies were measured for the first time in the acute phase and remission of the first relapse. In acute phase, severe ADAMTS13 deficiency $(<6 \%)$ in presence of antibodies was detected. We also measured protease level in the samples collected on the first day of clinical and laboratory remission which was achieved after 12days of treatment (normal neurologic status, platelet count $176 \times 10^{9} / \mathrm{L}, \mathrm{LDH} 447 \mathrm{IU} / \mathrm{L}$, haptoglobin $0.34 \mathrm{~g} / \mathrm{L}$, hemoglobin $106 \mathrm{~g} / \mathrm{L}$ ) and found ADAMTS 13 activity to be $24 \%$ in absence of antibodies. In remission 2 months after attainment of complete response, ADAMTS13 activity was found to be normal $(160 \%)$ with negative antibodies. The disease-free interval between the initial episode and the first relapse was 84 months. The interval between the first and second relapse was 7.5 months and between second and third relapse was 6months. In second relapse, clinically she presented with abdominal pain, purpura and alterations of urinalysis (proteinuria and hemoglobinuria). Laboratory examinations were as follows: hemoglobin $122 \mathrm{~g} / \mathrm{L}$ with 2-3 schistocytes in blood smear and $1.7 \%$ reticulocytes, platelets $8 \times 10^{9} / \mathrm{L}, \mathrm{LDH} 1454 \mathrm{U} / \mathrm{L}$, undetectable haptoglobin. She was treated with 16 daily PEX. Plasma volume exchange per each procedure was 1.5-2 circulatory volume. Cryosupernatant plasma was used as replacement fluid. She received pulse dose of methylprednisolone for
3 days, followed by per os prednisone. Vincristine was given in 2 doses of $1 \mathrm{mg}$ each and IV Ig in a total of 5 doses of $400 \mathrm{mg} / \mathrm{kg}$ each. Clinical presentation of third relapse was the same with laboratory parameters as follows: hemoglobin $124 \mathrm{~g} / \mathrm{L}$ with 6-7 schistocytes in blood film and $1.2 \%$ reticulocytes, platelets $12 \times 10^{9} / \mathrm{L}, \mathrm{LDH} 1381 \mathrm{U} / \mathrm{L}$, haptoglobin $0.92 \mathrm{~g} / \mathrm{L}$ and hemoglobinuria, erythrocyturia, proteinuria, leukocyturia and presence of bacteria's in urine. Treatment consisted of 14 daily PEX with 1.5 volume exchange using cryosupernatant plasma as replacement fluid. Additional therapy comprised of corticosteroids and IV $\operatorname{Ig}(25 \mathrm{~g} /$ day for 5 days $)$. ADAMTS13 parameters at the time of these other relapses were not measured.

Patient $n^{0} 5$, was first seen in her first relapse which was manifested exactly 11 years after initial episode. One year preceding first relapse she suffered of cerebrovascular insult without any criteria for TTP. Initial episode was treated with standard therapy consisted of corticosteroids and 5 single volume PE followed by FFP transfusion. She had fever of unknown etiology 3 days before relapse occurred. Complete remission was achieved with standard treatment. In acute phase of her first relapse, ADAMTS13 activity was moderately deficient $(11 \%)$ with no presence of specific antibodies. In remission, she remained with moderate ADAMTS13 deficiency $(20 \%)$ in absence of antibodies. The clinical course and the laboratory findings of patient are strongly suggestive of a late-onset congenital form of TTP. This condition is due to mutation on the ADAMTS13 gene. To confirm such a diagnosis it would be necessary to sequence the ADAMTS13 gene of patient.

Patient $n^{0} 14$, 17-year old female, experienced her first episode of TTP. Her elder sister in the age of 16 had episode of pulmonary embolism of unknown etiology. In clinical presentation, she had jaundice and lots of consciousness for 30seconds. Laboratory examination revealed severe thrombocytopenia $\left(12.7 \times 10^{9} / 1\right)$, hemoglobin $103 \mathrm{~g} / \mathrm{L}$, undetectable haptoglobin, LDH $2745 \mathrm{IU} / \mathrm{L}$, reticulocytes $9.3 \%, 4-5$ schistocytes per high-power field in peripheral blood smear and normal coagulation status. Due to inexperienced with this syndrome, attending pediatric physician's team didn't suspected patient as having highly probable TTP. Patient was given with IV Ig in dose $20 \mathrm{~g} /$ day for 2 days. Diagnosis was established on the $8^{\text {th }}$ hospitalization day after patient manifested clinical deterioration with unconsciousness lasting about 2 hours and sensomotor aphasia. PEX was commenced by 1volume exchange once a day (total number 5) but afterwards augmented by daily 1.5 volume exchange regimen (total number 16). Consolidation treatment consisted of 2 PEX season with single volume exchange followed by FFP transfusion for 4 consecutive days. Concomitantly with PEX, methylprednisolone was given in doses $500 \mathrm{mg}$ plus $1 \mathrm{~g}$ plus $1 \mathrm{~g}$ for 3 days, followed by prednisolone in dose $2 \mathrm{mg} / \mathrm{kg}$ until remission was achieved, with gradual reduction for 2 weeks thereafter. ADAMTS13 testing in acute phase revealed severe autoimmune protease deficiency, whereas in remission ADAMTS13 activity was normalized (61\%) with persistent antibodies. Over the subsequent 3.5months, the patient had 2 relapses. First relapse occurred 2.5 months after initial episode triggered by viral infection. Laboratory examination showed moderate thrombocytopenia and anemia (platelet count $78 \times 10^{9} / \mathrm{L}$, hemoglobin $113 \mathrm{~g} / \mathrm{L}$, LDH 517 IU/L, reticulocytes $1.88 \%$, no visible schistocytes). Patient had a prompt response to 4 daily double volume exchange. Three additional post-remission exchanges were performed. Patient didn't receive corticosteroids and other immune therapies. ADAMTS13 activity measured again in acute phase on second occasion and results showed severe protease deficiency in presence of specific antibodies. Second relapse occurred 1 month after first relapse with laboratory 
features as follows: platelet count $39 \times 10^{9} / \mathrm{L}$, hemoglobin $108 \mathrm{~g} / \mathrm{L}$, LDH 525U/L, reticulocytes $4.5 \%$, undetectable haptoglobin and no visible schistocytes. Patient was treated with 9 double volume PEX followed by 3 consolidation double volume PEX, prednisone $1 \mathrm{mg} / \mathrm{kg}$ and acetylsalicylic acid $100 \mathrm{mg} /$ day. ADAMTS13 parameters were not measured at the time of second relapse.

Patient $n^{0} 16$, 33- year old obese (weight $118 \mathrm{~kg}$, height $174 \mathrm{~cm}$ ) female, was first seen in her first relapse which had been developed 6years after initial episode of idiopathic TTP. Her initial episode was associated with 38 gestation week of her first pregnancy, when she was suffering from influenza. ADAMTS13 parameters were not measured at the time of pregnancy. She underwent section caesarean delivering a live healthy infant. After delivery, TTP was suspected and clinically diagnosed. Thus, she was treated with 3 single volume PEX and FFP transfusion for 7days. First relapse was triggered by viral infection of upper respiratory tract. PEX was withheld because of mild clinical presentation (platelet count $80 \times 10^{9} / \mathrm{L}$, hemoglobin $115 \mathrm{~g} / \mathrm{L}$, LDH 733IU/L) as well as an initial response to FFP transfusion and corticosteroid therapy. Complete remission was achieved for 3days. It was shown that patient had severe autoimmune ADAMTS13 deficiency in acute phase, which was normalized in remission (97\%) in presence of antibodies.

Pregnancy is thought to be in causal association to the presenting episode of TTP or HUS in $10-25 \%$. But, it is not clear whether acquired TTP-HUS is really initiated by pregnancy or coincidence with pregnancy reflects the fact that majority of affected women are in the age between 30 and 40years. Moreover, ADAMTS13 levels decrease in a second and third trimester as a physiological response during pregnancy (may be related to the physiological increase of von Willebrand factor concentration). Physiological protease decrement may be as low as $25-30 \%$ of the normal range. Additionally, multiple risk factors associated with pregnancy may provoke an acute episode of TTP-HUS in susceptible women. These include obesity and changes in hemostatic system (elevated plasma levels of fibrinogen, factor VIIa, factor VIII, von Willebrand factor, loss of endothelial cell thrombomodulin as well as diminishing of fibrinolytic activity) that progressively occur during the course of pregnancy, with the greatest hypercoagulable abnormalities occurring in term of delivery and immediately postpartum. ${ }^{17,19-21}$ It has been suggested that recurrent TTP with a subsequent pregnancy is uncommon. ${ }^{2}$ Taking into account that our patient $\mathrm{n}^{0} 16$ was obese and exposed to infection during pregnancy when she experienced her initial episode, at the entry of this study in absence of pregnancy she has been considered as having idiopathic TTP. We strongly adviced her for the necessity of the body mass reduction. None of the patients who had relapses died of the disease.

\section{Uncomplicated pregnancy/delivery secondary to previous episode among our idiopathic TTP patients}

In this cohort, there were 20female. 4/20 female patients $\left(n^{0} 14,18,23,24\right)$ were not pregnant before/after their original episodes. $15 / 20$ female had uncomplicated pregnancies/deliveries before initial episode. Ten of them $\left(\mathrm{n}^{0} 1,4,5,6,8,12,13,19,20,21\right)$ had 2 pregnancies/ deliveries while five of them $\left(n^{0} 2,9,15,17,22\right)$ had 1 pregnancy/ delivery before first episode. In one patient $\left(\mathrm{n}^{0} 16\right)$ initial episode was associated with 38 gestation week of her first pregnancy. Following initial TTP episodes, two patients ( $\mathrm{n}^{0} 4$ and 5) had 1 uncomplicated pregnancy and artificial abortion in the first trimester of their pregnancies each. Two of our patients defined with idiopathic TTP (cases $\mathrm{n}^{0} 2$ and $\mathrm{n}^{0} 17$ ) experienced initial TTP episodes when they had not been pregnant. Subsequently, they had uncomplicated pregnancy and delivered with a live, healthy infants. On the whole, in this cohort only one patient experienced TTP episode related to pregnancy.

Patient $n^{0} 2$, 27-year old female presented with her initial idiopathic TTP episode seven days after upper respiratory tract infection. Laboratory data were as follows: platelet number $14 \times 10^{9} / \mathrm{L}$, hemoglobin $89 \mathrm{~g} / \mathrm{L}$, LDH $4559 \mathrm{IU} / \mathrm{L}$, reticulocytes $5 \%$, schistocytes $5 \%$, undetectable haptoglobin. Clinical picture was characterized by abdominal pain, vomiting, headache and fever. Complete remission was achieved with 5daily double volume PEX with concomitant corticosteroids administration. Consolidation was performed with 2 PEX session followed by FFP transfusion for 3days. ADAMTS13 activity was $40 \%$ in acute phase. Protease levels were tested in remission on two occasions in two years period and activities were $100 \%$ and $130 \%$, respectively. Five years after initial episode she had uncomplicated pregnancy and at term vaginal delivery of a live, healthy male infant weighing $3.5 \mathrm{~kg}$.

Patient $n^{0}$ 17, 30-year old woman, presented with initial TTP episode, associated with a 4-days history of prolonged menstrual bleeding, bruising, fever, jaundice, symptomatic anaemia and at the time of admission she had hallucinations. The patient's laboratory parameters were as follows: platelet count $11 \times 10^{9} / \mathrm{L}$, hemoglobin $78 \mathrm{~g} / \mathrm{L}$, reticulocytes $19 \%$, schistocytes $4-5$ in blood film, undetectable haptoglobin, LDH 3709IU/L. She was treated with PEX as well as intravenous methylprednisolone for 3days followed by orally prednisone in a dose $1 \mathrm{mg} / \mathrm{kg}$. Remission was achieved with 5 PEX with 1.5 volume exchange. Consolidation treatment consisted of 1 PEX with 1,5volume exchange, followed by FFP transfusion in dose $15 \mathrm{~mL} / \mathrm{kg}$ for 5 days. ADAMTS13 activity was severely reduced at the time of acute episode and normalized (93\%) in absence of antibodies in remission. Five years ago, in her first pregnancy there were no complications related to TTP. One year after initial episode she had uncomplicated second pregnancy. She underwent section caesarean at term delivering a live healthy female infant weighing $3.8 \mathrm{~kg}$. Preceding delivery, platelet number was $265 \times 10^{9} / \mathrm{L}$ and remained within similar range for days after delivery. She didn't receive any blood product.

\section{Discussion}

Recurrent episodes are usually prompt diagnose based on the presence of stringent diagnostic criteria consisted of thrombocytopenia and fragmented anemia in absence of the signs of organ damage that are considered to be relatively late events that should be avoided by urgent treatment ${ }^{5}$. Recurrences of TTP are generally milder than first episode. It has been reported about different clinical severity of first episode and recurrences of TTP in a 51 patients with two or more TTP episodes ${ }^{1}$. The results of this study showed that platelets count and hemoglobin levels were higher in recurrences than in the first disease episode and LDH levels were lower with mild or no clinical manifestation. This could be explained by timely diagnose and treatment. Although almost recurrent episodes of TTP are timely recognizable by both patient and physician, there is potential for diagnostic difficulty pertains to the occasional patients who present with neurological deficit and other signs of tissue ischemia before developing overt thrombotic microangiopathy. ${ }^{22}$ In our patient $\mathrm{n}^{0} 7$, the third disease episode was indistinguishable from the initial presenting one as well as the first relapse. Namely, an acute myocardial infarction was a presenting sign of the second TTP relapse whereas the degree of thrombocytopenia as well as elevation of LDH were less severe 
than in former episodes. Haptoglobin level was normal accompanied with hemoglobin value around $130 \mathrm{~g} / \mathrm{L}$. This emphasize that recurrent episodes of TTP may be clinically more severe than previous one and should always be regarded as serious clinical condition with potentially fatal outcome. In clinical practice, defining of remission remains a clinical determination relies on laboratory measure such as hematological recovery, normalization of LDH and resolution of clinical signs. Therefore, it is plausible that some recurrences with apparent clinical induction of remission are actually a continuum of active subclinical disease. ${ }^{9}$ In our patient $\mathrm{n}^{0} 14$, short time intervals between recurrences ( 1 and 2.5 months, respectively) may suggest that disease was altered but not resolved.

Recurrences occurred with a spectrum of presentations ranging from a single relapse to several episodes developing with variable frequency. In Shumak et al. ${ }^{6}$ study, 63patients who recovered from an acute episode of TTP were analyzed to determine the incidence and timing of relapses during clinical follow up ranged from 3 to 10years. One or more episodes of relapse were documented in $17 / 63(27 \%)$ patients, occurring 7 months to 8 years after the original episode. In our cohort, after entry to study 2 patients had relapse only once, 2patients relapsed two times and 1 patient had three recurrent episodes. Of them, 2 patients have had relapses within 1year. We saw $7 / 17(41,17 \%)$ idiopathic TTP patients with recurrent disease. Vesely et al. ${ }^{12}$ first reported about association of obesity with TTP-HUS. In their prospective cohort of 142patients diagnosed with TTP or HUS, half of patients with severe ADAMTS13 deficiency were obese. They reported about obesity as an additional contributing risk factor for the development of TTP-HUS by causing endothelial dysfunction, enhancing tissue factor expression or causing low- grade systemic inflammation. In our cohort, 2patients who experienced relapses were obese. It seems clear that idiopathic TTP caused by ADAMTS13 deficiency tends to relapse and relapses are usually associated with persistent or recurrent ADAMTS13 deficiency.

In 109 patients who survived an acute episode of acquired TTP, in the frame of a retrospective cohort study, Peyvandi at al..$^{15}$ evaluated the predictive role of ADAMTS13 as measured in plasma during remission. They found that survivors with severely reduced levels of ADAMTS13 and/or with anti-ADAMTS13 antibodies during remission have an approximately three-fold greater likelihood of developing another episode than patients with higher protease activity and no antibody. The search for predictors of TTP recurrence was also the focus of a study by Ferrari et al., ${ }^{16}$ who investigated 32patients who had low plasma levels of ADAMTS13 activity at the time of first acute episode of TTP and subsequently achieved remission. Persistence of low ADAMTS13 activity at remission was a predictor of recurrence, which occurred in 6/32 (19\%) patients using an 18-month follow-up. According to The Oklahoma TTP Registry ${ }^{10}$ data for survivors with initial episode of clinically diagnosed TTP in whom ADAMTS13 activity was measured, relapse rate was significantly more frequent among survivors with ADAMTS13 activity $<10 \%$ $(16 / 47,34 \%)$ than among survivors with ADAMTS13 activity of $10 \%$ or more $(5 / 136 ; 4 \%)$. Among the 136 surviving patients with ADAMTS13 levels of $10 \%$ or more, relapses occurred within 2 years. Among the 47 survivors with ADAMTS13 activity $<10 \%, 10(63 \%)$ initial relapses occurred within the first year and 14(88\%) within 4years. The remaining 2 patients had their initial relapses at 6.4 and 7.5 years. The estimate of the cumulative risk for relapse at 7.5 years among patients with ADAMTS 13 levels $<10 \%$ was $41 \%$. Concerning the recurrences of syndrome, the main finding in this cohort of patients was occurrence in patients with idiopathic TTP which was characterized by ADAMTS13 deficiency in acute phase. Furthermore, severe autoimmune ADAMTS13 deficiency was determined in all cases who relapsed with the exception of 2 patients (case $n^{0} 4$ with partial protease deficiency in presence of antibodies in acute phase and normal protease activity with persistent antibodies in remission and case $\mathrm{n}^{0} 5$ with partial protease defect in absence of antibodies in acute phase of first relapse as well as in remission).

\section{Conclusion}

Recurrences of TTP syndrome occurred with a spectrum of presentations ranging from a single relapse to several episodes developing with variable frequency. It seems clear that idiopathic TTP caused by ADAMTS13 deficiency tends to relapse and relapses are usually associated with persistent or recurrent ADAMTS13 deficiency. The relatively less severe clinical course of recurrences should be taken into account when potentially harmful preventive therapies are being planned in patients considered at risk for TTP recurrence.

\section{Acknowledgements}

None.

\section{Conflict of interest}

The author declares no conflict of interest.

\section{References}

1. Lotta LA, Mariani M, Consonni D, et al. Different clinical severity of first episodes and recurrences of thrombotic thrombocytopenic purpura. Br J Haematol. 2010;151(5):488-494.

2. Sadler JE, Moake JL, Miyata T, et al. Recent advances in thrombotic thrombocytopenic purpura. Hematology Am Soc Hematol Educ Program. 2004;2004:407-423.

3. George JN. How I treat patients with thrombotic thrombocytopenic purpura-hemolytic uremic syndrome. Blood. 2000;96(4):1223-1229.

4. Moake JL. Thrombotic thrombocytopenic purpura. In: Kitchens CS, et al. editors. Consultative Hemostasis and Thrombosis. 3rd ed. Saunders, UK; 2007. p. 405-420.

5. Sadler JE. Von Willebrand factor, ADAMTS13, and thrombotic thrombocytopenic purpura. Blood. 2008;112(1):11-18.

6. Shumak KH, Rock GA, Nair RC. Late relapses in patients successfully treated for thrombotic thrombocytopenic purpura. Canadian Apheresis group. Ann Intern Med. 1995;122(8):569-572.

7. Bandarenko N, Brecher ME. United states thrombotic thrombocytopenic purpura aphresis study group (US TTP ASG): Multicenter survey and retrospective analysis of current efficacy of therapeutic plasma exchange. J Clin Apher. 1998;13(3):133-141.

8. Bell WR. Thrombotic thrombocytopenic purpura/hemolytic uremic syndrome relapse: frequency, pathogenesis, and meaning. Semin Hematol. 1997;34(2):134-139.

9. Willis MS, Bandarenko N. Relapse of thrombotic thrombocytopenic purpura: is it a continuum of disease? Semin Thromb Hemost. 2005;31(6):700-708.

10. Hovinga JAK, Vesely SK, Terrell DR, et al. Survival and relapse in patients with thrombotic thrombocytopenic purpura. Blood. 2010;115(8):1500-1511.

11. Zheng XL, Kaufman RM, Goodnough LT, et al. Effect of plasma exchange on plasma ADAMTS13 metalloprotease activity, inhibitor level and clinical outcome in patients with idiopathic and nonidiopathic thrombotic thrombocytopenic purpura. Blood. 2004;103(11):4043-4049. 
12. Vesely SK, George JN, Lämmle B, et al. ADAMTS13 activity in thrombotic thrombocytopenic purpura-hemolytic uremic syndrome: relation to presenting features and clinical outcomes in a prospective cohort of 142 patients. Blood. 2003;102(1):60-68.

13. Raife T, Atkinson B, Montgomery R, et al. Severe deficiency of VWF-cleaving protease (ADAMTS13) activity defines a distinct population of thrombotic microangiopathy patients. Transfusion. 2004;44(2):146-150.

14. Coppo P, Bengoufa D, Veyradier A, et al. Severe ADAMTS13 deficiency in adult idiopathic microangiopathies defines a subset of patients characterized by various autoimmune manifestation, lower platelet count, and mild renal involvement. Medicine (Baltimore). 2004;83(4):233-244.

15. Peyvandi F, Lavoretano S, Palla R, et al. ADAMTS13 and anti-ADAMTS13 antibodies as markers for recurrence of acquired thrombotic thrombocytopenic purpura during remission. Haematologica. 2008;93(2):232-239.

16. Ferrari S, Scheiflinger F, Rieger M, et al. Prognostic value of anti-ADAMTS13 antibody features (Ig isotype, titer and inhibitory effect) in a cohort of 35 adult French patients undergoing a first episode of thrombotic microangiopathy with undetectable ADAMTS13 activity. Blood. 2007;109(7):2815-2822.
17. Allford SL, Hunt BJ, Rose P, et al. Guidelines on the diagnosis and management of the thrombotic microangiopathic hemolytic anaemias. $\mathrm{Br} J$ Haematol. 2003;120(4):556-573.

18. Yamaguchi M, Nagata K, Hamaguchi H. Successful treatment of refractory thrombotic thrombocytopenic purpura with vincristine: a case report. Haema. 2005;8:300-303.

19. George JN, Vesely SK. Thrombotic thrombocytopenic purpura-hemolytic uremic syndrome: Diagnosis and treatment. Cleve Clin $\mathrm{J} \mathrm{Med.}$ 2001;68(10):857-864.

20. Scully M, Starke R, Lee R, et al. Successful management of pregnancy in woman with a history of thrombotic thrombocytopaenic purpura. Blood Coagul Fibrinolysis. 2006;17(6):459-463.

21. George JN. The association of pregnancy with thrombotic thrombocytopenic purpura-hemolytic uremic syndrome. Curr Opin Hematol. 2003;10(5):339-344.

22. Downes KA, Yomtovian R, Tsai HM, et al. Relapsed thrombotic thrombocytopenic purpura presenting as an acute cerebrovascular accident. $J$ Clin Apher. 2004;19(2):86-89. 